\title{
Nghiên cứu của Trung tâm ISR, Trường Đại học Phenikaa được trích dẫn trong báo cáo chính thức của ISI
}

\author{
Hồ Mạnh Toàn \\ Trung tâm Nghiên cứu Khoa học Xã hội liên ngành \\ Trường Đại học Phenikaa \\ Hà Đông, Hà Nội, Việt Nam
}

Phenikaa University, 09/10/2019; ( ${ }^{\text {URL: http://phenikaa-uni.edu.vn/chitiet/tin-tuc- }}$ truong/nghien-cuu-cua-trung-tam-isr-truong-dai-hoc-phenikaa-duoc-trich-dan-trongbao-chinh-thuc-cua-isi-2019-10-9-2-58-262)

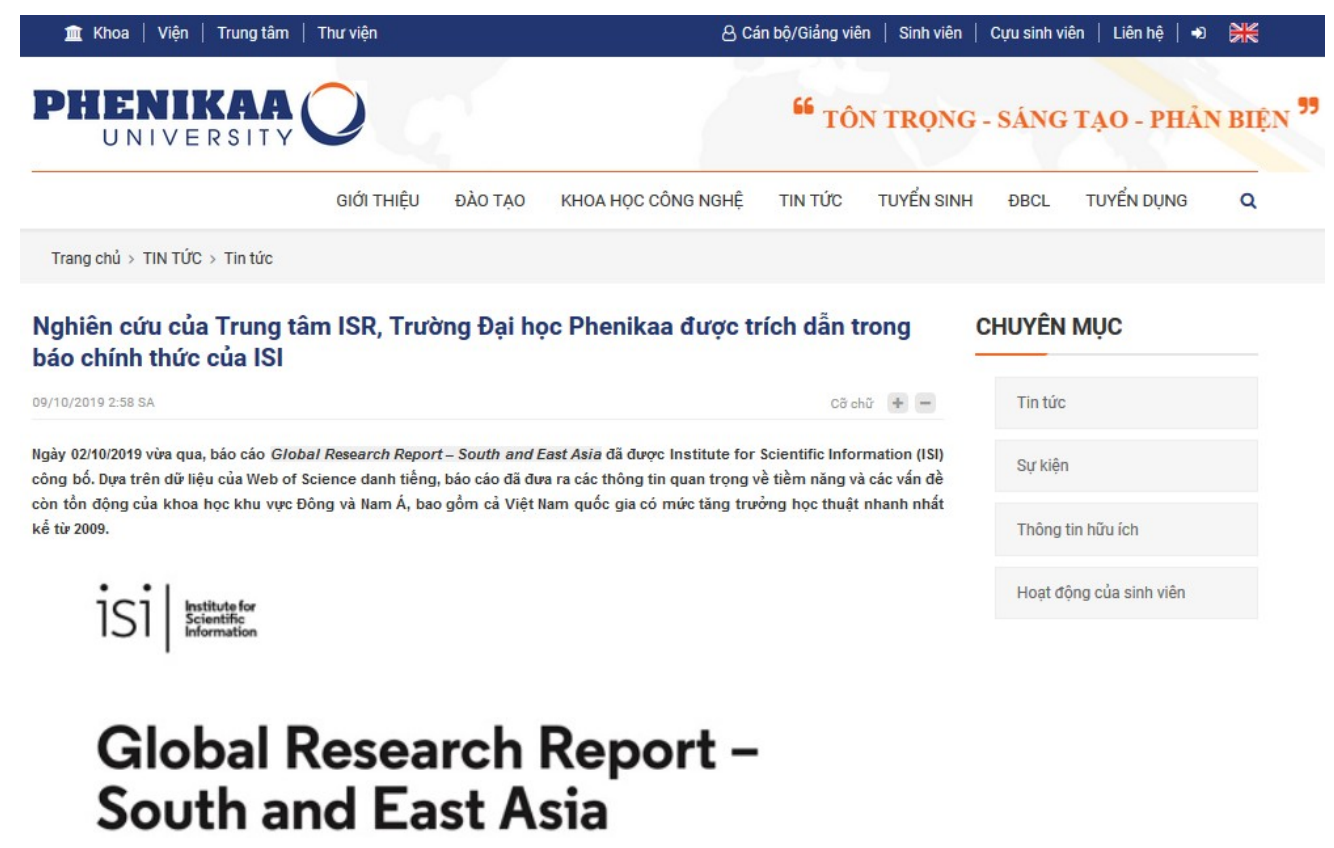

(Ảnh: Website trường Đại học Phenikaa, 9/10/2019)

Ngày 02/10/2019 vừa qua, báo cáo Global Research Report - South and East Asia đã được Institute for Scientific Information (ISI) công bố. Dựa trên dữ liệu của Web of Science danh tiếng, báo cáo đã đưa ra các thông tin quan trọng về tiềm năng và các vấn 
đề còn tồn đọng của khoa học khu vực Đông và Nam Á, bao gồm cả Việt Nam quốc gia có mức tăng trưởng học thuật nhanh nhất kể từ 2009.

\section{isi| \\ Global Research Report - South and East Asia}

\section{Professor Jonathan Adams, David Pendlebury, Gordon Rogers \& Dr Martin Szomszor}

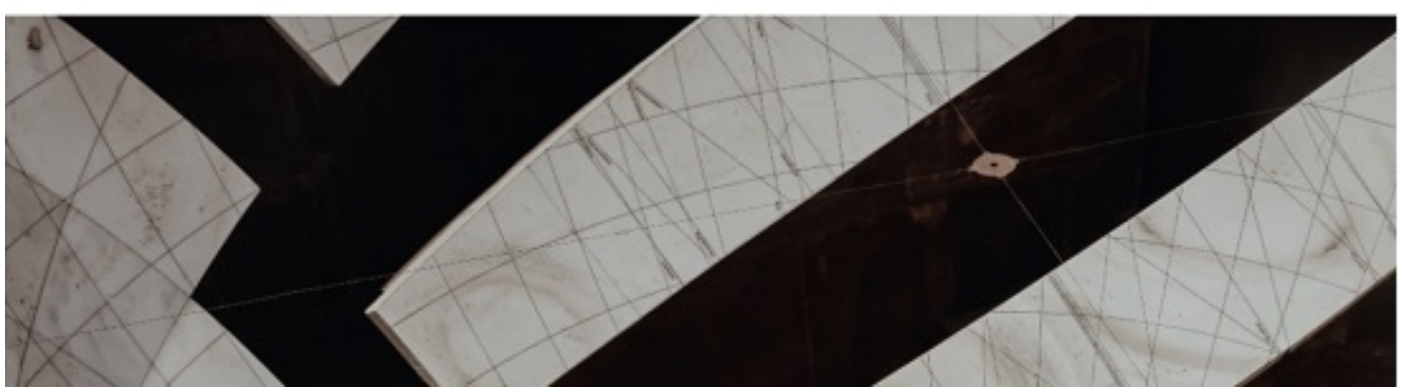

(Ảnh bìa báo cáo Global Research Report - South and East Asia của ISI)

Nội dung báo cáo ngay sau đó đã được các tờ báo lớn trong nước như báo điện tử Vietnamnet đưa tin vào ngày 08/10/2019, chỉ 2 ngày trước Lễ kỷ niệm 12 năm thành lập trường Đại học Phenikaa.

\footnotetext{
vietnamnet.vn/vn/giao-duc/khoa-hoc/bao-cao-nghien-cuu-toan-cau-tai-nam-va-dong-nam-a-viet-nam-tang-truong-nhanh-nhat-khu-vuc-574891.html

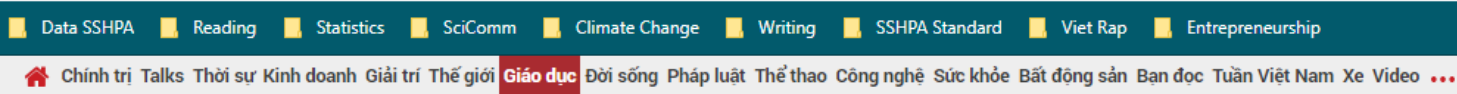

Nguyen T V, Ho-Le TP and Le U V. (2017). International collaboration in scientific research in Vietnam: an analysis of patterns and impact. Scientometrics, 110, 1035-1051

Nguyen TV and Pham L T. (2011). Scientific output and its relationship to knowledge economy: an analysis of ASEAN countries. Scientometrics, 89, 107-117.

Phuong T T, Duong H B and McLean G N. (2015). Faculty development in Southeast Asian higher education: a review of literature. Asia Pacific Education Review, 16, 107-117.

Vuong $Q$ H. (2019). The harsh world of publishing in emerging regions and implications for editors and publishers: The case of Vietnam. Learned Publishing, 32(4), 314-314; DOI: 10.1002/leap. 1255
}

(Ảnh trích báo điện tử Vietnamnet, 8/10/2019) 
Để giúp độc giả hiểu thêm về khoa học tại các quốc gia Đông và Nam Á, báo cáo của ISI cũng đã gợi ý các nghiên cứu, bài viết nổi bật về khoa học tại các quốc gia trong báo cáo. Mặc dù chỉ mới được công bố vào tháng 7-2019, nhưng bài viết solo về khuynh hướng phát triển và tương lai của xuất bản học thuật tại Việt Nam (DOI:

10.1002/leap.1255) của TS Vương Quân Hoàng (Trung tâm ISR, trường Đại học Phenikaa) trên tạp chí Learned Publishing [2018 JIF = 2.2; CiteScore = 1.89], đã nhanh chóng được các tác giả của báo cáo Global Research Report - South and East Asia đưa vào danh sách gợi ý cùng với các nghiên cứu khác về Việt Nam xuất bản từ các năm 2011 (DOI: 10.1007/s11192-011-0446-2), 2015 (DOI: 10.1007/s12564-015-9353-1) và 2017 (DOI: $10.1007 / \mathrm{s} 11192-016-2201-1)$.

Tạp chí Learned Publishing thuộc hiệp hội Association of Learned and Professional Society Publishers (ALPSP) (https://www.alpsp.org/) và do Wiley xuất bản, tập trung vào biên tập, xuất bản và truyền thông hàn lâm, hướng tới các cá nhân đang hoạt động chuyên nghiệp trong lĩnh vực này.

Theo SCImago, tạp chí Learned Publishing được xếp hạng Q1 trong lĩnh vực Truyền thông (Communication), với $\mathrm{H}$-Index $=28$. Ấn phẩm này có IF 2.2 (mức rất cao đối với ngành biên tập khoa học, và nằm trong Top $15 \mathrm{H}$-Index của Google Metrics trong phân mục Library \& Information Science:

https://scholar.google.com/citations?view op=top venues\&hl=en\&vq=soc libraryinfor mationscience).

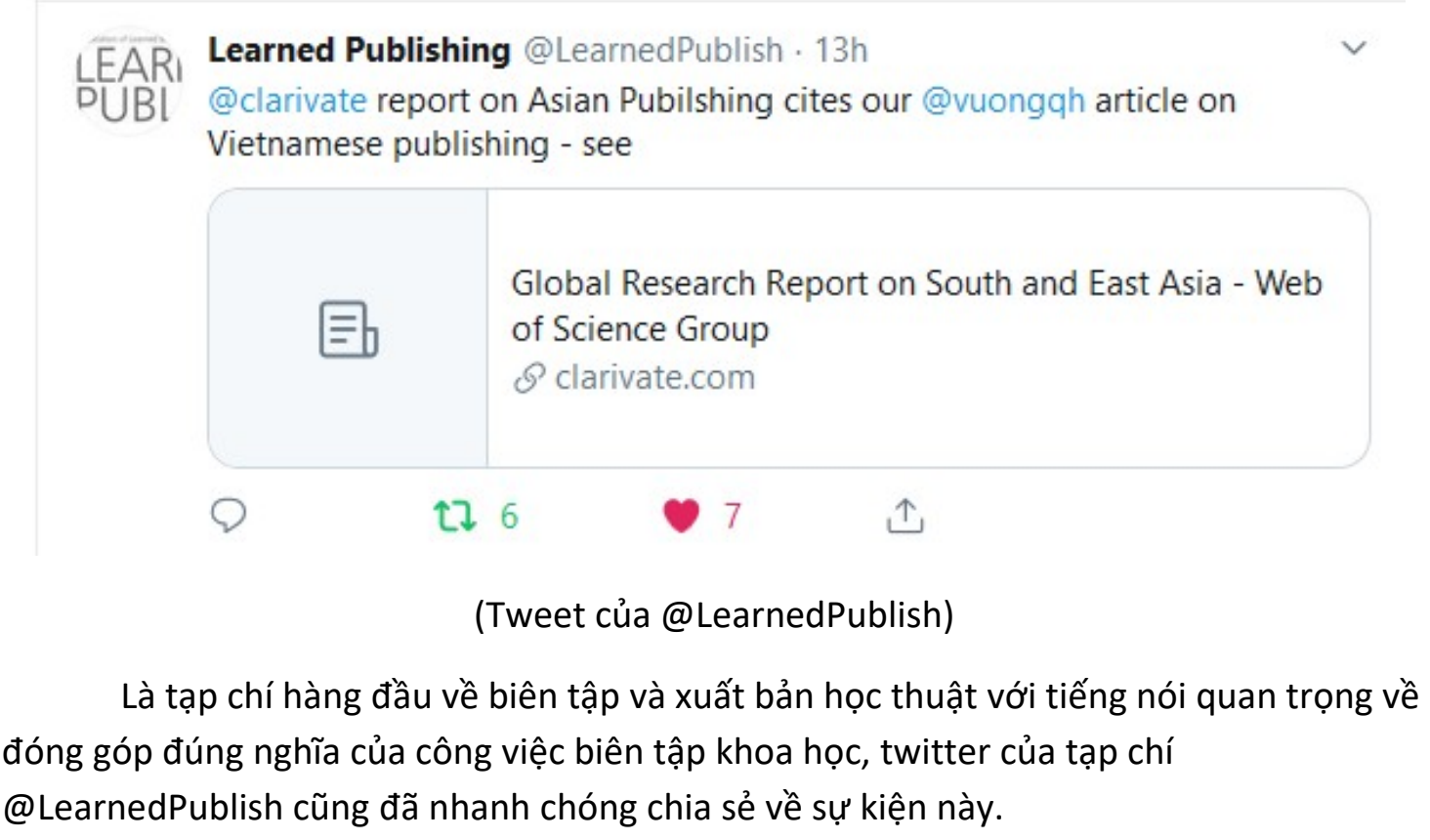




\section{Tài liệu tham khảo:}

Ho, M. T., \& Pham, H. (2019, Oct. 8). Việt Nam tăng trưởng nhanh nhất khu vực Nam và Đông Nam Á về công bố khoa học. Vietnamnet. Retrieved from:

https://vietnamnet.vn/vn/giao-duc/khoa-hoc/bao-cao-nghien-cuu-toan-cau-tainam-va-dong-nam-a-viet-nam-tang-truong-nhanh-nhat-khu-vuc-574891.html. (accessed: Oct. 8, 2019).

Nguyen T. V., Ho-Le, T. P., \& Le, U. V. (2017). International collaboration in scientific research in Vietnam: an analysis of patterns and impact. Scientometrics, 110, 1035-1051.

Nguyen, T. V., \& Pham, L, T. (2011). Scientific output and its relationship to knowledge economy: an analysis of ASEAN countries. Scientometrics, 89, 107-117.

Phuong, T. T., Duong, H. B., \& McLean, G. N. (2015). Faculty development in Southeast Asian higher education: a review of literature. Asia Pacific Education Review, 16, 107-117.

Vuong, Q. H. (2019). The harsh world of publishing in emerging regions and implications for editors and publishers: The case of Vietnam. Learned Publishing, 32(4), 314324. 